Supporting Information

\title{
Oxygen Isotopic Substitution of Peptidyl Phosphates for Modification-Specific Mass Spectrometry
}

\author{
Yu Shi and Xudong Yao* \\ Department of Chemistry, University of Connecticut, Storrs, CT 06269 \\ *: Corresponding author. Email: x.yao@uconn.edu
}



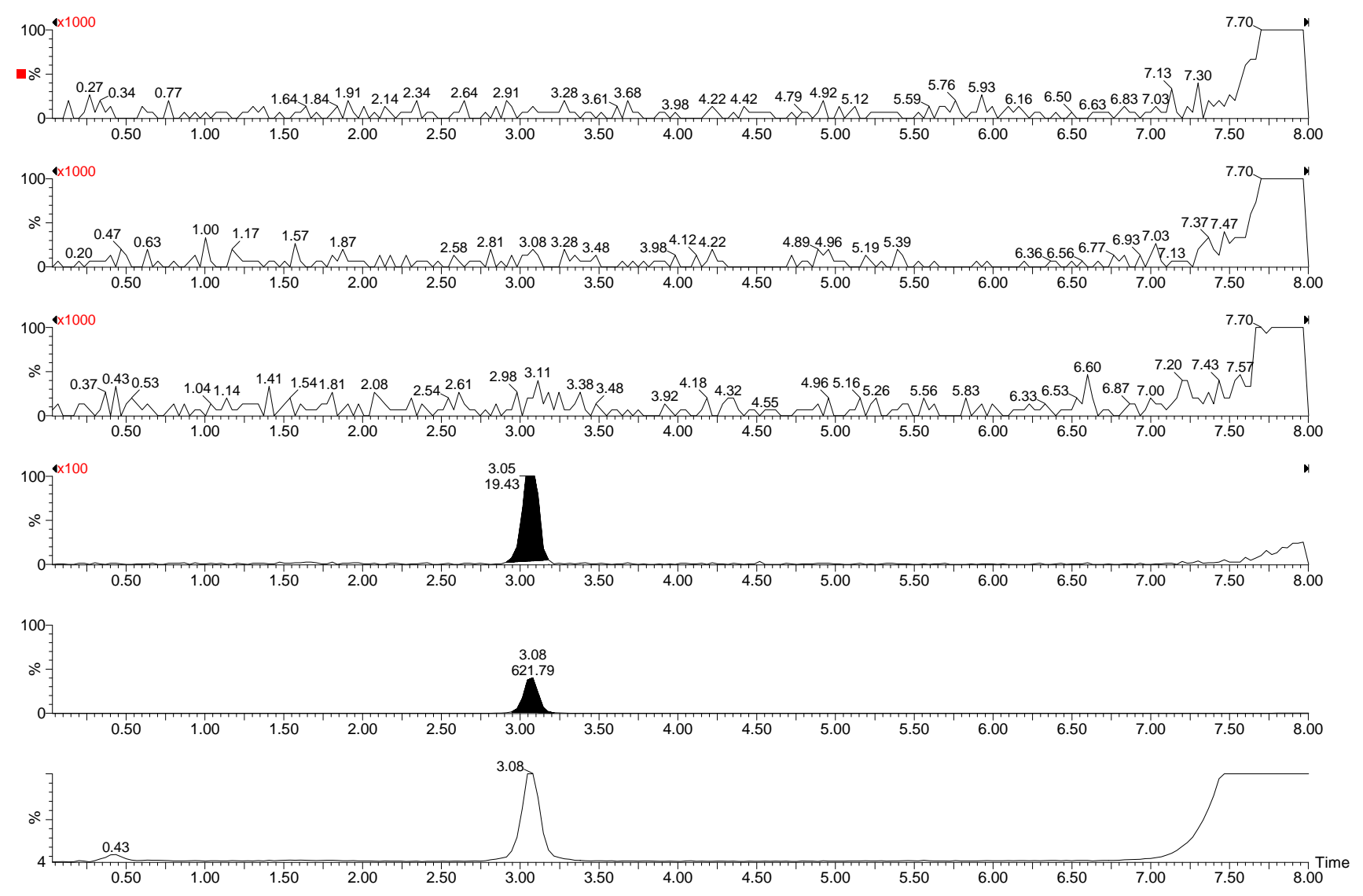

Figure S1. Efficiency for the methylation reaction of carboxylate groups on insulin receptor

phosphopeptide. From the bottom to the top are the total ion chromatogram for the reaction mixture, the extracted ion chromatogram for the permethylated peptide, the extracted ion chromatogram for the peptide with one un-methylated carboxylate group (magnified by 100 times), the extracted ion chromatogram for the peptide with two un-methylated carboxylate groups (magnified by 1000 times), the extracted ion chromatogram for the peptide with three un-methylated carboxylate groups (magnified by 1000 times), and the extracted ion chromatogram for the peptide with four un-methylated carboxylate groups (magnified by 1000 times). Y-axes are normalized. Although there is detectable signal for the extracted ion chromatogram for the peptide with one un-methylated carboxylate group, it is likely not a partial methylation product, but the gas-phase fragment from the permethylated peptide ions, because they have the same elution time. There are no detectable products with carboxylate groups un-methylated. 

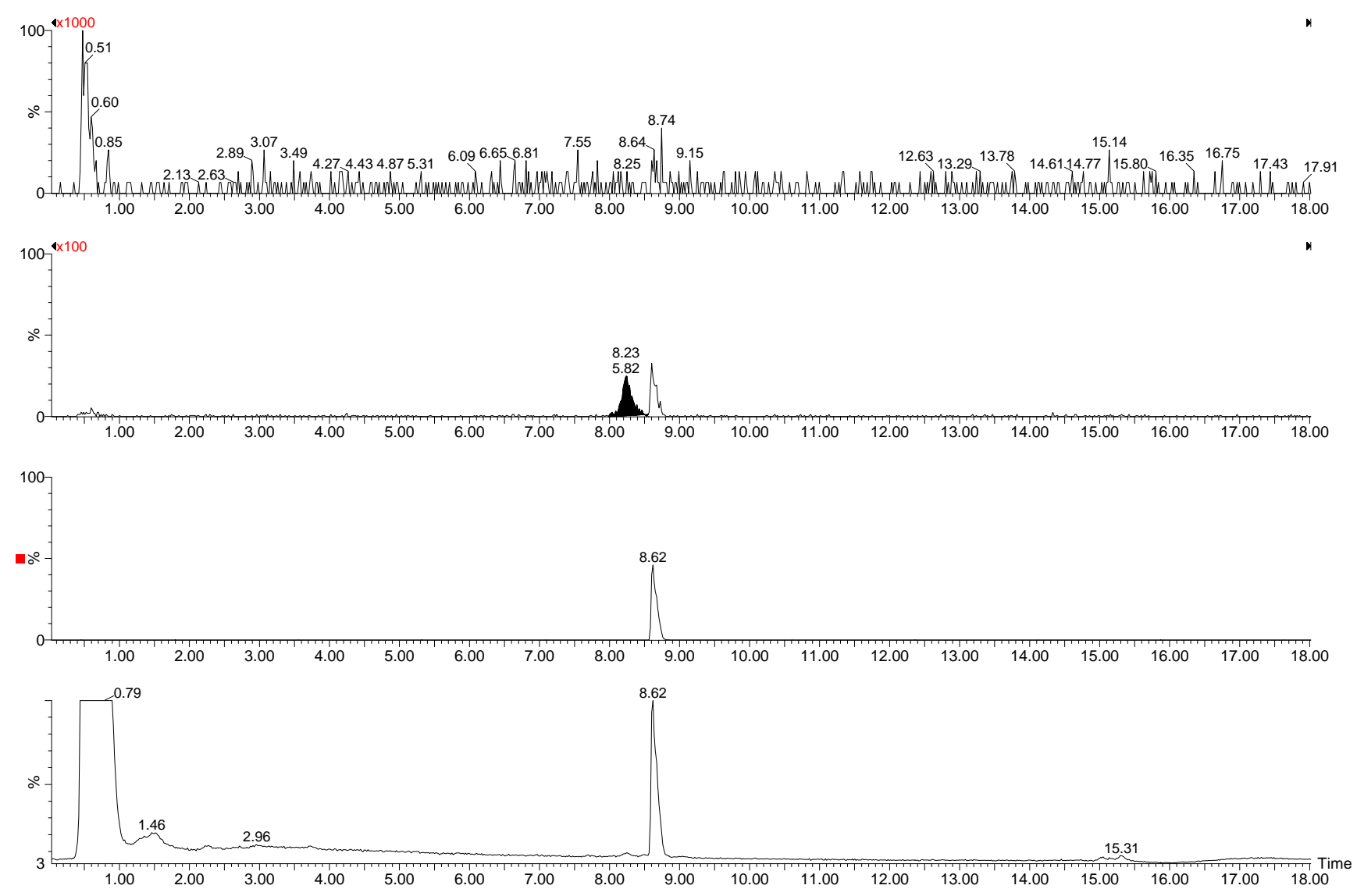

Figure S2. Efficiency for carbodiimide-assisted formation of the phosphoramidate of the permethylated insulin receptor phosphopeptide. From the bottom to the top are the total ion chromatogram for the reaction mixture, the extracted ion chromatogram for the fully amidated product (three phosphoramidates), the extracted ion chromatogram for the product with one unreacted phosphate group (magnified by 100 times), and the extracted ion chromatogram for the product with two unreacted phosphate groups (magnified by 1000 times). Y-axes are normalized. For the extracted ion chromatogram for the product with one unreacted phosphate group, there are two peaks. The first one is the true partial reaction product (less than $1 \%$ of the fully amidated product). The second peak represents the gas-phase fragment ion of the fully amidated product. There is no detectable product with either one or zero phosphate group amidated. 


\section{Group 1 Group 2 Group 3}

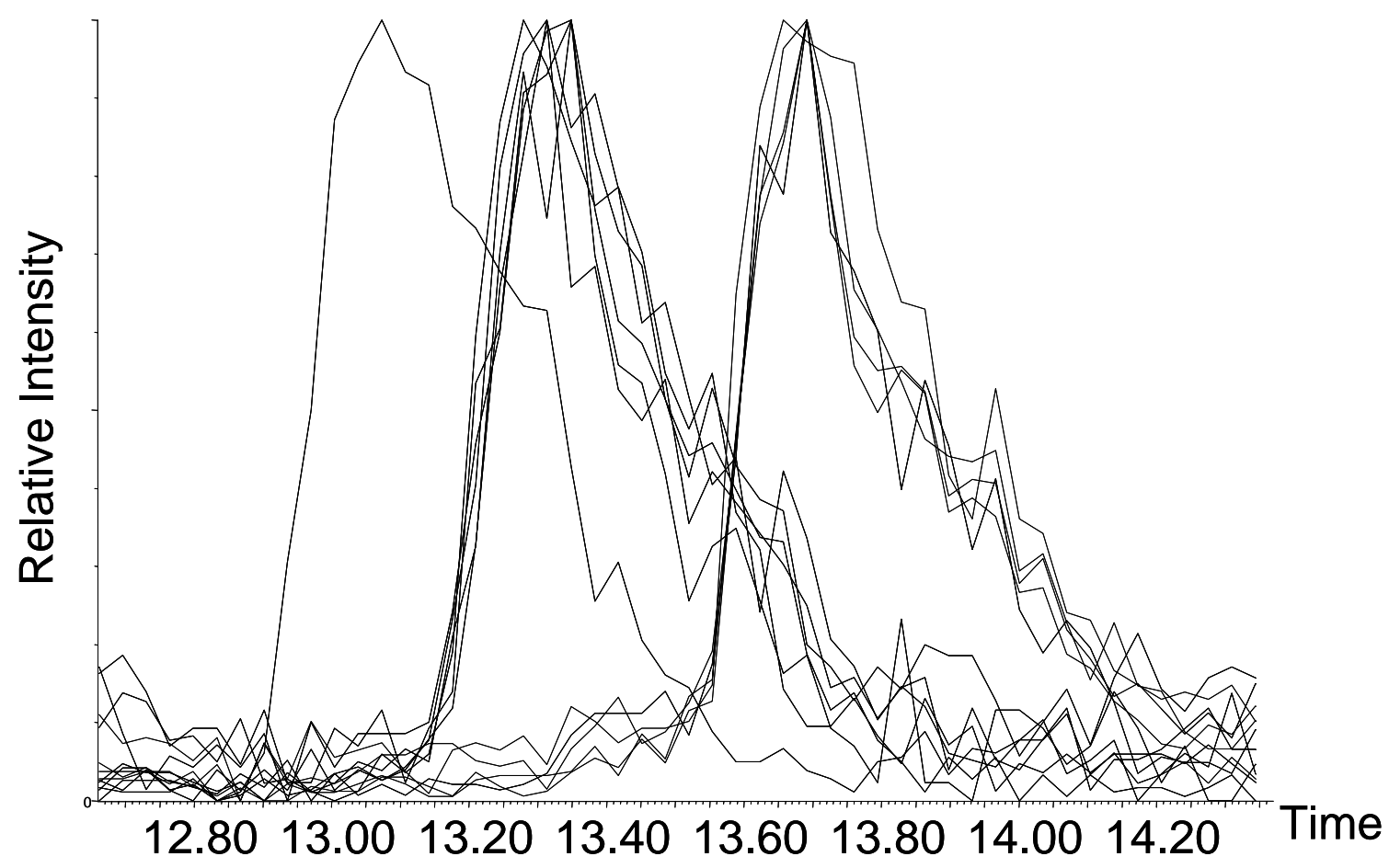

Figure S3. Correlation of precursor and fragment ions of the tryptic NPF peptide (SpYEEHIP) with the differentially labeled immonium ions of pY. Two pools of a nonphosphoprotein were spiked with peptide NPF (KRSpYEEHIP). They were reduced, alkylated, and then digested by trypsin. The resulted peptide mixtures were subjected to the labeling procedure described in the manuscript in parallel, one using $\mathrm{H}_{2}{ }^{16} \mathrm{O}$ and the other using $\mathrm{H}_{2}{ }^{18} \mathrm{O}$. These two pools of peptide mixtures were combined for LC-p ${ }^{2}$ CID mass spectrometry. We observed both 216.043 and 218.047 maker ions (with elution time of $13.3 \mathrm{~min}$ ), as well as several a- and b-ions for the digested NPF peptide (SpYEEHIP). These ions showed in doublets indicating the differentially labeling on the phosphate group. Extracted ion chromatograms for these ions superposed to form peaks in Group 2. Group 3 contained the extracted ion chromatograms for several singlet ions. Group 2 overlapped with Group 3, as well as Group 1, in agreement with the observation that the ions in these groups co-existed in the time window between 13.1 to $13.8 \mathrm{~min}$. However, these ions could be sorted into three groups based on different peak elution time. 